improve. These figures indicate that the danger period is the second year, the third year, and the first half of the fourth year of life, the figures for these periods being under the general average of the complete series. These results are in keeping with the known epidemiological

\begin{tabular}{|c|c|c|c|c|}
\hline Age of Patients & $\begin{array}{l}\text { Total } \\
\text { Cases }\end{array}$ & $\begin{array}{c}\text { No } \\
\text { Attack }\end{array}$ & $\begin{array}{l}\text { Per cent. } \\
\text { Protected }\end{array}$ & $\begin{array}{l}\text { Per cent. } \\
\text { Average of } \\
\text { Three } \\
\text { Groups }\end{array}$ \\
\hline Months 0-2 & - & - & - & - \\
\hline $3-5$ & 29 & 38 & 97.43 & - \\
\hline $6-8$ & 19 & 18. & 94.74 & 94.74 \\
\hline $9-11$ & 18 & 16 & 88.89 & 85.45 \\
\hline One year $0-2$ & 18 & 13 & 72.22 & $\varepsilon 0.85$ \\
\hline 3- 5 & 11 & 9 & 81.82 & 69.56 \\
\hline $6-8$ & 17 & 10 & 58.82 & 73.53 \\
\hline $9-11$ & 6 & 6 & 100 & 72.73 \\
\hline Two years $0-2$ & 21 & 16 & 76.19 & 75 \\
\hline $3-5$ & 5 & 2 & 40 & 75.68 \\
\hline $6-8$ & 11 & 10 & 90.91 & 75 \\
\hline $9-11$ & 4 & 3 & 75 & 76.92 \\
\hline Th:ee years 0-2 & 11 & 7 & 63.63 & 72.22 \\
\hline $3-5$ & 3 & 3 & 100 & 68.75 \\
\hline $6-8$ & 2 & 1 & 50 & 83.33 \\
\hline 911 & 1 & 1 & 100 & 90.91 \\
\hline Four years 0- 2 & 8 & 8 & 100 & 100 \\
\hline $3-5$ & - & - & - & 100 \\
\hline $6-8$ & 6 & 6 & 100 & 100 \\
\hline 911 & 1 & 1 & 100 & - \\
\hline Total & 201 & 168 & 83.58 & - \\
\hline
\end{tabular}

facts that the first five years of life are those most susceptible. The figures make it clear that the effect of serum injection closely follows the same curve.

4. Donors of Serum.-Again it is difficult to evaluate the serum, as the other factors exert some influence on the result. Comparison of the tables compiled during the investigation show that, while as a whole the serums of the donors were fairly potent, there were undoubtedly some poor ones. For instance, in one case of crossinfection twelve contact patients were injected, and only three remained free from attack. The serum used was from the same donor injected in varying doses, but the effect produced was poor in comparison with that obtained under less favourable circumstances with another serum. Possibly, had another donor been chosen the results would have been more favourable, as the records indicated that this particular donor had had a less severe measles attack than the average.

5. Amount of Serum. Injected.-Little can be said on the practical side, since the dose given in this series (5 c.cm.) was practically constant. In one instance, however, varying doses were given on the sixth day, and it was found that while $3.4 \mathrm{c.cm}$. gave either no attack or attenuation, a dose of 1.7 c.cm. gave resulting mild attacks.

6. Duration of Immunty. - The duration of the immunity produced by the serum varied in many of the cases; it probably varies with the amount injected. In my series, using the standard dose of 5 c.cm., modified cases occurred after the usual incubation period, indicating that the protection afforded was not always complete. The number of secondaries is probably larger than that recorded, but judging from the figures available in my series the number is greatest in those injected in the first six days of exposure. This seems to suggest that the period of complete protection given by $5 \mathrm{c.cm}$. is not much longer than fourteen days, but, as can be seen from the " tertiary" infections, it may extend up to six weeks, and even then the protective power is still sufficient to modify the attack.

\section{General Conclusions}

Clinically there was a striking difference in the type of attack after such a small dose as $5 \mathrm{c.cm}$., even up to the eighth or tenth day of exposure. Of all the patients who developed measles after serum not one was really ill, and, in fact, many would not have been reported had not the slightest appearance of any sign been noted as evidence of infection. Generally speaking, it would appear that the best method of utilizing the serum is not to prevent measles by large doses, but rather to cause what might be a fatal attack to be an attenuated one with no complications. The active immunity thus produced will be invaluable, whereas the passive immunity lasts only two to six weeks in the complete state.

Summarizing the results obtained, there is no doubt that the injections were of great benefit, as they secured complete protection in almost $\mathbf{8 0}$ per cent. That this did not always result suggests that if such is the object a sufficient dosage should be employed on an early day after exposure. The useful period for complete protection in my series appears to be slightly greater than other investigators have found, but it must be remembered that the suspicion of measles was sufficient to mean the immediate removal of a possible source of contagion from children's wards, resulting in a comparatively short period of exposure. Administratively, the procedure of protective inoculation of convalescent serum was of great value in controlling possible outbreaks of measles in hospitals and country" homes devoted to the treatment of young children.

\section{ISOLATED DEXTROCARDIA}

BY

T. H. G. SHORE, M.D., F.R.C.P.

PHYSICIAN TO THE SOUTH DEVON AND EAST CORNWALL HOSPITAL, PLYMOUTH

Although reports on a considerable number of cases of isolated dextrocardia have been published, the condition is sufficiently rare to justify recording another case.

Dextrocardia naturally accompanies complete transposition of the viscera, which is not a very uncommon condition. All the organs are then transposed to the opposite side of the body, and at the same time their individual forms are transposed, the result being a mirror-image of the normal. I have seen several examples of this condition during life and one post mortem. Rarely, such a transposition is incomplete so that one organ or more escapes ; a few instances have been recorded of transposition of all the organs except the heart. In the following case the heart only was transposed to the right side.

If displacements resulting from disease are excluded, true dextrocardia is a developmental error. According to Lichtman, ${ }^{1}$ who has made a most careful review of the subject, most of the recorded cases have died in the first year of life; if the patient lives to the age of 10 the expectation of life is 30 years, after 20 the expectation is 44 years. Few, however, are so fortunate.

The subject when studied in detail is much more complicated than would at first appear. Dextrocardia, as it occurs with complete transposition-a mirror-image both 
in position and in the relation of the chambers to one another-would seem to be a rare form of a rare condition. More often the chambers are not arranged as they would be in a mirror-image. Thus the right or venous ventricle may lie on the right side, and form the apex of the heart, whereas in a mirror-image transposition the apex would be formed by the arterial ventricle, corresponding to the left in the normal left-sided position. In such a case the chambers bear the normal relation to one another, though the heart as a whole is transposed. Again, in either of these forms of dextrocardia the aorta may pass over the right or left bronchus, descending through the thorax on the left side of the vertebral column. When the right aortic arch persists in this way the aorta passes behind the oesophagus to reach the other side. Four possibilities must therefore be recognized: a mirror-image transposition with right or left aortic arch, and a transposed heart with normally related chambers with right or left aortic arch. A centrally placed heart, with the venous ventricle to the right forming the apex, is a recorded stage towards one of the last two conditions.

By far the greater number of cases of isolated dextrocardia are the subjects of other congenital malformations of the heart, the most common being defects of the interventricular or interauricular septa. Conditions occur which may be regarded as derivatives of this primary defect-namely, one functioning and one " aborted" ventricle, or a trilocular heart. Such cases as these seldom reach adult life. Lichtman has traced 161 cases in the literature, of which only three were free of other congenital abnormality when studied clinically and post mortem ; all were of the type having normally related chambers. $\mathrm{He}$ states that so far only four cases have been studied by all the methods that can now be employed: clinically, by $x$ rays, by the electrocardiograph, and post mortem.

\section{Case Record}

The patient, a carpenter aged 42, complained of pain over the left side of his chest, which had been noticed for about ten years. He had joined the Navy in 1910, having passed a medical examination in Devonport. In 1922 he was invalided out of the Service for heart trouble, and about that time the pain began. The pain comes on in attacks after exertion and radiates to the left arm, which then becomes powerless; the attack lasts, with decreasing severity, for the remainder of the day. The area previously painful is then tender to the touch, although lately this has not been so apparent. Between the attacks the patient is breathless on exertion, and sometimes he has swelling of the feet at night, especially if he has been at work. Recently there have been frequent fainting or giddy attacks on first rising from bed in the morning. There is no history of rheumatism.

\section{Clinical Examination}

On examination the man was of slight build, with some pallor, but no cyanosis or oedema. His pulse was regular, and varied between 60 and 76 ; respirations between 18 and 24 ; temperature was always slightly subnormal. The apex beat was not palpable, but the cardiac dullness extended three inches to the right of the mid-line and up to the third rib on the right side ; the other border lay just to the left of the left sternal margin. There was a decided bulging of the chest wall over an area rather larger than that of the cardiac dullness. The heart sounds were best heard to the right of the sternum, and consisted of a soft, toneless, blowing murmur and a fairly normal second sound. The murmur was loud just to the left of the sternum, but was not conducted far, nor to the vessels of the neck; it was heard loudly over the area of cardiac dullness. The systolic blood pressure was $140 \mathrm{~mm}$., diastolic pressure $95 \mathrm{~mm}$. No other abnormality was found in the body. The Wassermann reaction was strongly positive.

\section{$X$-Ray Examination}

The heart lies definitely to the right side, its position corresponding to that found on clinical examination. The heart is rather more globular in outline than normal; this was especially seen in the rounded apex. The aortic knuckle was clearly shown in its normal left-sided position, indicating that the aorta passes over the left bronchus. The left side of the diaphragm lies at a slightly higher level than the right, and the liver and stomach occupy their normal positions. In the first oblique position one of the auricles could be seen projecting backwards into the mediastinum ; this is the transposed left (arterial) auricle, which in the normal position of the heart lies posteriorly. Screen examination showed that the right and left borders of the cardiac shadow contract synchronously, so the left border must be composed of venous ventricle, and must be considerably enlarged. The venous auricle occupies a higher position than usual, and does not participate in the formation of this border of the heart.

\section{EIECTROCARDIOGRAPH}

The rhythm is regular, the rate 69 . The upright stroke in Lead 1 seems at first sight to be $R$, and to be followed by inversion of $\mathrm{T}$. If, however, the distance from the beginning of this wave to the end of $T$ be measured it will be found equal to the distance from the end of $R$ (beginning of $S$ ) to the end of $T$ in other leads. It is therefore an inverted $S$ wave, and the whole of Lead 1 is inverted. S in Lead 1 is enlarged. The height of $\mathrm{R}$ in Lead 2 is greater than that in Lead 3. If these two leads are interchanged, as would be the case in a mirror-image dextrocardia in complete transposition of viscera, then the position is enlargement of $S$ in Lead 1 and of $\mathrm{R}$ in Lead 3 , which is what is found when the right side preponderates in a normally situated heart. In view of the transposition the electrocardiogram must be taken to indicate that there is a preponderance of the venous ventricle. (Fig.1.)

A very simple way of checking these observations, and one which might very well be employed as a routine in cases of doubt, is to accommodate the electrocardiograph to the patient by transposing the arm leads and taking the foot lead from the right instead of the left foot. This removes the factor of the patient's transposition and enables the interpretation

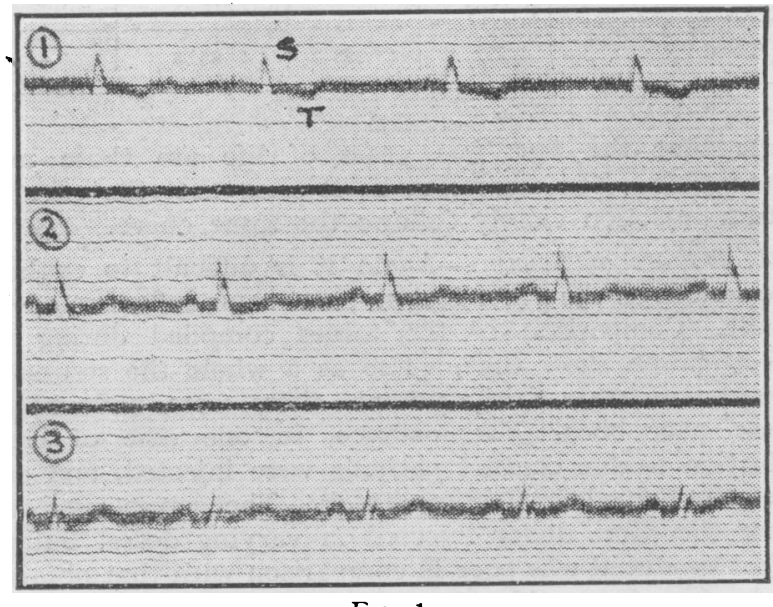

FIG. 1.

to be made as if his heart were normally placed. In this case the result of the manœuvre, shown in Fig. 2, is clearly to indicate preponderance of the right (venous) side in a series of curves which are easy to internret.

This case is, in all probability, one of mirror-image dextrocardia, with preponderance of the venous ventricle. The aorta crosses the left bronchus, so that the heart only is transposed. The clinical and $x$-ray examinations render this much of the diagnosis clear, but the electrocardiograph gives information as to the direction of the conduction path, and consequently to the probable arrangement of the chambers of the heart. The correct interpretation of the electrocardiogram depends upon the recognition of the upstroke in Lead 1 as $\mathrm{S}$, inverted and enlarged, and this can only be done by direct measurement, and confirmed by reversing the leads to the instrument. On reading some of the descriptions already 
published confusion becomes apparent in the interpretation of the upright wave in Lead 1. Its nature does not seem to have been defined by direct measurement, but several of the tracings shown are demonstrably capable of the same interpretation as is given here, and in all probability reversal of the leads would have dismissed all doubt.

Preponderance of the right side is to be expected in view of the murmur, which is almost certainly of congenital origin, and most likely indicates a septal defect.

Parsons Smith, in recording two similar cases during the war, considered that their early cardiac breakdown was brought about through the relation of the right-sided heart to the solid liver; instead of to the more yielding

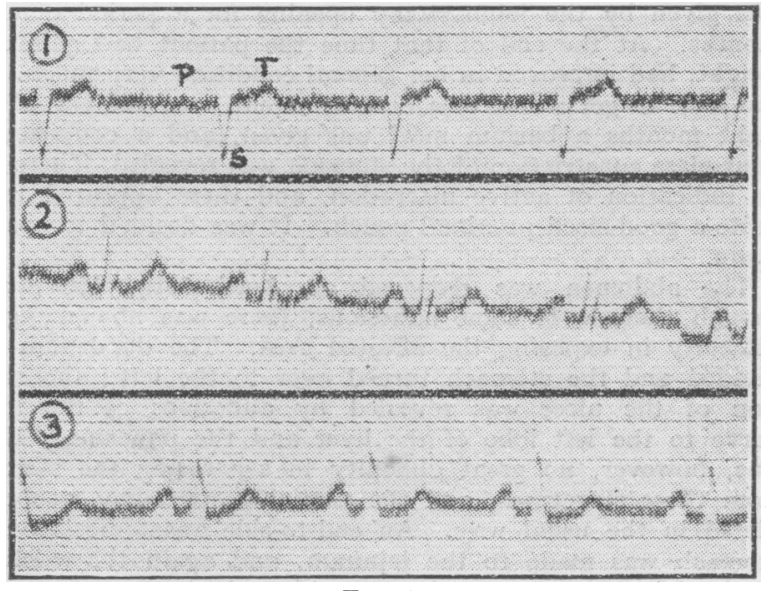

Fig. 2.

stomach, this hampering the physiological dilatation on exertion. Such effect was not produced in persons who had complete transposition of their viscera, because the heart was then related to the stomach. This is very likely the case, and it is therefore the more remarkable that this man was able to serve in the Navy for twelve years, including the war period, before his heart broke down.

The complaint of pain on exertion, involving the left chest and arm, is of interest. Pain due to disease of the right coronary artery, or to infarction of its area of supply, may be felt on the right side of the chest and down the right arm in a person whose heart is normally situated. In this case the distribution is reversed, presumably because the venous ventricle from which it originates is transposed.

The notes of this case are recorded by the kind permission of Dr. C. D. Lindsay, under whose care the patient was admitted to the South Devon and East Cornwall Hospital in June, 1932.

Bibliography

${ }^{1}$ Lichtman, S. S.: Arch. Int. Med., 1931, xlviii, 683, 866

This paper gives 370 references to this and allied conditions. For the convenience of those who cannot readily consult foreign papers the following list of those more recently published in this country are given, but Lichtman's paper is by far the most comprehensive:

Abrahamson, L.: Quart. Journ. Med., 1924-5, xviii, 335.

Aitken, A.: Brilish Medical Journal, 1917, ii, 428.

Aitken, A.: Brilish Medral Journal, 1917, 11, 428.

Brooks, N. P.: Med. Record, 1911, lxxix, 962.
Capon, N. B., and Chamberlain, N.: Lancet, 1925, i, 918.

Carpenter, G.: Brit. Journ. Child. Dis., 1904, i, 160.

Feldman, W. NI: Ibid., 1925, xxii, 136

Ferguson, V. I.: British Medical Journal, 1928, ii, 894.

Graham, J. H. P.: Journ. R.A.M.C., 1906, vii, 498.

-rossage: Trans. Clin. Soc. Lond., 1901, xxxiv, 220.

Hawthorne, C. O.: British Medical Journal, 1907, i, 1186.

Jones, H. W.: Ibid., 1924, i, 147.

Keith, A.: Lancet, 1909, ii, 359, 433, 519

Keith, A.: Lancet, 1909, ii, 359, 433, 519.
Krestin, D.: British Medical Journal, 1927, ii, 1223.

Mrestin, D.: British Medical Journal,

MacLennan, W.: Ibid., 1896, ii,

IcLaughlin, A. I. G.: Lancet, 1924, ii, 1064

Owen, S. A.: Ibid., 1912, ii, 113.

Parsons Smith, B.: Ibid., 1919, ii, 1076

Tate, R. G..H.: Journ. R.A.M.C., 1907, viii, 434.

Whipham, T. R.: Proc. Roy. Soc. Med., 1909, ii, 169.

\section{JEJUNOSTOMY IN THE TREATMENT OF MASSIVE GASTRIC ULCER}

\author{
BY
}

O. STANLEY HILLMAN, M.S., F.R.C.S.

ASSISTANT SURGEON, ROYAL PORTSMOUTH HOSPITAL; CONSULTING SURGEON, PETERSFIELD COTTAGE HOSPITAL

The treatment of simple ulcers of the body of the stomach was discussed at a meeting of the Surgical Section of the Royal Society of Medicine in July, 1931. There was, however, no definite pronouncement made on the value of jejunostomy in the treatment of cases of the more " massive" and difficult type. The extent of any opinion with regard to jejunostomy was indicated by $\mathrm{Mr}$. GordonTaylor in the following words: "For the extensive ' letter-box,' or inaccessible, ulcer I have little experience and less success with jejunostomy." 1 At about the time that this statement was made I had had two cases which demonstrated in such a striking and convincing way the value of jejunostomy that I feel constrained to record them as a small contribution to this subject.

\section{CASE I}

A man, aged 38, who had been invalided frcm the Navy on account of his gastric ulcer, first came under my care in October, 1930. He ccmmenced to have symptoms of a gastric ulcer in 1924, his pain coming on two hours after food. The condition progressed in the usual way, with intermissions of freedom, until he had to enter a naval hospital in July, 1929. After six weeks of "alkali treatment" he had a small haematemesis. In September, 1929, a posterior gastrojejunostomy was performed, which, in a subsequent skiagram taken after a barium meal, was seen to be functioning. The operation afforded only temporary relief, and in July, 1950 , he had a severe haematemesis, was subsequently invalided out of the Service, and referred to me at the Royal Portsmouth Hospital by his civilian doctor.

When I first saw him he was having constant attacks of pain, and was able to eat very little food: he had marked anaemia, and looked very ill. A barium meal revealed that no food was passing through the stoma, but that all of it went through the pylorus, which was deformed. Operation was advised. He was admitted to hospital on January 7 th, 1931, having had continuous pain, from which he could get no relief, for a month. He was put on strict medical treatment in the hope of improvement, but nothing would relieve his pain, and so, in spite of his bad general condition, it was decided that operative treatment must be attempted as the only possible hope of recovery.

On January 14th the abdomen was opened under high percaine spinal anaesthesia and gas and oxygen. Great difficulty was experienced from the beginning on account of dense adhesions to the previous operation wound, so that much time was taken up in revealing the condition of the viscera. Eventually, there was found to be a large inflammatory mass, which involved the lower half of the stomach, the transverse colon, and a loop of jejunum. With considerable care this mass was freed, and it was found that anything like a radical operation would have meant the resection of a portion of the stomach, transverse colon, and jejunum-a very formidable undertaking. At this point the patient became very collapsed, and any operation of such a nature was quite out of the question; consequently the mass was replaced and a jejunostomy performed, care being taken to leave plenty of jejunum above the opening to allow of a subsequent Polya type of partial gastrectomy. The jejunostomy tube was brought through the left rectus muscle and the abdomen closed. The patient made a very gocd recovery from this operation, and all feeding was carried out through the jejunostomy. As he still had some gastric pain, alkalis were given by the mouth for a short time, but soon these were discarded; nothing at all was then given by the mouth, and he complained of no more pain. With the absence of pain and with the increase in nourishment that, the jejunostomy afforded, the improvement was marked; but still 